ISSN electrónico: 2445-1355

DOI: https://doi.org/10.14201/fj2021621725

\title{
EVOLUCIÓN DE LA DISPENSACIÓN DE ESPECIALIDADES FARMACÉUTICAS ESTUPEFACIENTES DESDE EL AÑO 2000 AL 2020 EN LA OFICINA DE FARMACIA SITUADA EN LA PLAZA DEL MERCADO CHICO DE LA CIUDAD DE ÁVILA
}

\section{Evolution of the dispensing of narcotic pharmaceutical specialties from 2000 to 2020 in the pharmacy office located in the Plaza del Mercado Chico in the city of Avila}

Carlos PÉREZ JIMÉNEZ*; Paloma CARVAJAL GALLARDO**; Carmen GARCÍA MARTÍN***

*Farmacia Juan Carlos Pérez Antona; *Farmacia Carvajal Gallardo; **Farmacia José Sahagún Krause. Calle Mártires de El Tiemblo 32. 05270 El Tiemblo (Ávila). Correo-e: carvagalla@usal.es**

RESUMEN: Se registraron año por año todas las dispensaciones de especialidades farmacéuticas estupefacientes, sin discernir entre ellas, desde el año 2000 al 2020 en la farmacia de la plaza del Mercado Chico, 10, de Ávila. El objeto de este estudio es comprobar si existe alguna variación en cuanto a la dispensación de estas a lo largo de los años y de qué tipo es esa variación.

Se realizó el conteo y agrupación de los datos gracias a la declaración anual de estupefacientes de cada año, proporcionada por la farmacia y el correspondiente análisis de estos datos a través del programa informático Microsoft Excel.

Gracias a los resultados obtenidos se llegó a la conclusión de que realmente sí existía una variación en la dispensación de especialidades estupefacientes. 
CARLOS PÉREZ JIMÉNEZ; PALOMA CARVAJAL GALLARDO; CARMEN GARCÍA MARTÍN EVOLUCIÓN DE LA DISPENSACIÓN DE ESPECIALIDADES FARMACÉUTICAS ESTUPEFACIENTES DESDE EL AÑO 2000 AL 2020 EN LA OFICINA DE FARMACIA SITUADA EN LA PLAZA DEL MERCADO CHICO DE LA CIUDAD DE ÁVILA

Concretamente, hay una tendencia creciente en la dispensación de estas a medida que han ido pasando los años desde el 2000 al 2020.

Palabras clave: Estupefacientes; dispensación; Ávila; tendencia.

ABSTRACT: Beginning in the year 2000, all dispensing of narcotic pharmaceutical specialties is registered. This practice continued until the year 2020, including the pharmacy Plaza del Mercado Chico, 10, Ávila. The purpose of this study is to analyze whether there is any kind of variation when it comes to this dispensing over the years, and what that variation implies.

The data used to for this study was gathered through the annual declaration of narcotic drugs, which is released by the pharmacy for further analysis on Microsoft Excel.

The results of this study suggest that there exists a variation on the movement of narcotic drugs that have been dispensed. This implies that there has been an upward trend towards this dispensing of pharmaceutical specialties from 2000 to 2020.

Keywords: Narcotics; dispensing; Ávila; tendency.

\section{INTRODUCCIÓN}

Los estupefacientes son alcaloides naturales del opio y sus correspondientes derivados semisintéticos. Entre las acciones ejercidas por estos compuestos destaca la analgesia, ya que se debe a que alteran la percepción del dolor a nivel de la medula espinal y de los centros superiores del Sistema Nervioso Central. También pueden producir un efecto anestésico conseguido a dosis superiores y junto a algún coadyuvante.

El primer principio activo que se consiguió aislar fue la morfina. Su estructura química tridimensional consta de cinco anillos, tres de ellos se encuentran dispuestos en el mismo plano, otro contiene un nitrógeno y el otro anillo es perpendicular a los otros tres. A partir de este principio activo se obtuvieron análogos gracias a modificaciones realizadas en su estructura química, tales como oxidación, adición o deleción de grupos funcionales y reducción.

Por tanto, para que estos compuestos tengan actividad analgésica, es esencial que su estructura contenga un anillo aromático y un átomo de carbono cuaternario unido a una amina terciaria por otros dos átomos de carbono. 
CARLOS PÉREZ JIMÉNEZ; PALOMA CARVAJAL GALLARDO; CARMEN GARCÍA MARTÍN

EVOLUCIÓN DE LA DISPENSACIÓN DE ESPECIALIDADES FARMACÉUTICAS ESTUPEFACIENTES

DESDE EL AÑO 2000 AL 2020 EN LA OFICINA DE FARMACIA SITUADA EN LA PLAZA DEL MERCADO

CHICO DE LA CIUDAD DE ÁVILA

\subsection{Mecanismo de acción}

Los opioides, tanto endógenos como exógenos, se unen en el organismo a una serie de receptores específicos. De los más de diecisiete que se han llegado a describir destacan tres, todos relacionados con los receptores de membrana acoplados a proteína $\mathrm{G}$ (Valdivielso, 1998): los receptores mu $(\mu)$, que están relacionados con un efecto analgésico, eufórico, sedante y de depresión respiratoria; los kappa (К), que se relacionan con un mayor efecto analgésico y sedante e incluso de miosis, y los delta $(\delta)$, que se relacionan con una cierta analgesia y euforia.

$\mathrm{Al}$ interaccionar el opioide con el receptor, se inhibe la adenilatociclasa, disminuyendo la concentración de AMPc; además se produce la apertura de los canales de potasio y el cierre de canales de calcio, lo que hace que se reduzca la excitabilidad neuronal. Estas sustancias tienen efectos duraderos debido al aumento de los factores de transcripción CREB y $\Delta$ FosB (Álvarez, Farré, 2005).

Los opioides se van a clasificar, según su eficacia y afinidad por los receptores, en agonistas puros, parciales, agonistas/antagonistas y antagonistas.

\subsection{Farmacocinética}

Para muchos estupefacientes existen preparados de uso parenteral (intramuscular, intravenoso y subcutáneo), también por vía oral, vía transdérmica e incluso vía transnasal. En el ámbito de la oficina de farmacia, su administración se limita a la vía oral y transdérmica.

En cuanto a la vía oral, la mayoría de estupefacientes se absorben bien; aunque algunos presentan una biodisponibilidad por debajo del $50 \%$, debido a un importante efecto de primer paso hepático (Valdivielso, 1998).

En cuanto a la vía transdérmica, solo se administran opioides con elevada liposolubilidad y bajo peso molecular, para que así puedan alcanzar la circulación sistémica (Valdivielso, 1998).

Una vez absorbidos, se distribuyen rápidamente por el organismo, variando entre 1,5-4,7 L/Kg su volumen de distribución. La forma principal de inactivación de estos fármacos es el metabolismo hepático, el cual suele consistir en una oxidación microsomal y la conjugación con un ácido glucurónido (Álvarez, Farré, 2005).

La semivida de eliminación suele ser corta (2-4 horas). Se excretan sobre todo por la orina y por la bilis, sufriendo circulación enterohepática (Álvarez, Farré, 2005).

\subsection{Indicaciones}

Los estupefacientes destacan por su potente acción analgésica. Constituyen la base de los fármacos utilizados en el tratamiento de enfermedades que vienen 
CARLOS PÉREZ JIMÉNEZ; PALOMA CARVAJAL GALLARDO; CARMEN GARCÍA MARTÍN

EVOLUCIÓN DE LA DISPENSACIÓN DE ESPECIALIDADES FARMACÉUTICAS ESTUPEFACIENTES

DESDE EL AÑO 2000 AL 2020 EN LA OFICINA DE FARMACIA SITUADA EN LA PLAZA DEL MERCADO CHICO DE LA CIUDAD DE ÁVILA

acompañadas de dolor moderado/intenso de tipo agudo o de dolor crónico, más del tipo somatosensorial.

Hace años su uso era más limitado a enfermedades oncológicas, actualmente se usan de forma más frecuente en casos como: dolor postoperatorio, artritis, ciática, fracturas óseas, edema pulmonar, problemas oncológicos, disnea asociada a insuficiencia ventricular, etc.

En cuanto a dolores intensos asociados a inflamación o dolores de tipo neuropático (migrañas), el uso de opioides se ha comprobado que no resulta eficaz en su tratamiento.

\subsection{Interacciones}

- Las fenotiazinas potencian los efectos de los opioides.

- Los IMAOs inhiben la enzima CYP450 por la que se metabolizan los opioides, pudiendo estos causar toxicidad.

- Algunos antidepresivos tricíclicos actúan como inductores enzimáticos disminuyendo la eficacia de los opioides.

- Ciertos estudios afirman que el alcohol junto con estupefacientes aumenta considerablemente el riesgo de depresión respiratoria.

- Los barbitúricos potencian el efecto depresor de los opioides, pudiendo producir una sobredosis o importantes reacciones adversas.

- Los sedantes, anestésicos generales e bipnóticos están contraindicados junto al uso de estupefacientes debido a un aumento grave del efecto depresor sobre el SNC.

\subsection{Efectos adversos}

- Estreñimiento: los estupefacientes disminuyen los movimientos peristálticos intestinales causando cuadros severos de estreñimiento.

- Depresión respiratoria: a altas dosis, los analgésicos opioides pueden afectar al centro que regula la respiración causando una depresión respiratoria que puede llevar a la muerte.

- Dependencia y tolerancia: el uso continuado de estos puede llegar a producir el conocido síndrome de abstinencia si después se interrumpe su uso.

- Náuseas y vómitos.

- Somnolencia y sedación.

- A nivel psicológico: su uso puede causar alucinaciones, euforia, delirios, confusión, etc. 
CARLOS PÉREZ JIMÉNEZ; PALOMA CARVAJAL GALLARDO; CARMEN GARCÍA MARTÍN

EVOLUCIÓN DE LA DISPENSACIÓN DE ESPECIALIDADES FARMACÉUTICAS ESTUPEFACIENTES

DESDE EL AÑO 2000 AL 2020 EN LA OFICINA DE FARMACIA SITUADA EN LA PLAZA DEL MERCADO CHICO DE LA CIUDAD DE ÁVILA

- Prurito: debido al uso de los estupefacientes el nivel de histamina en el organismo es bajo, lo que puede producir erupciones en la piel.

- Miosis.

- Mioclonía.

\section{2. Овjetivos}

\section{Objetivo general:}

Comprobar si se produce una variación y qué tipo de variación en la dispensación de estupefacientes en dicha farmacia desde los años 2000 al 2020.

\section{Objetivos especificos:}

- Mejorar el conocimiento sobre el tratamiento del dolor y las especialidades farmacéuticas estupefacientes.

- Adaptar el estocaje de estupefacientes a la actualidad.

\section{Material y Metodos}

Se diseñó un estudio observacional de tipo retrospectivo, en el periodo que abarca desde el año 2000 al 2020, en la oficina de farmacia ubicada en la plaza del Mercado Chico, 10, en la ciudad de Ávila.

Esta se caracteriza por ser una farmacia de barrio que al estar en una zona muy céntrica su clientela habitual es de una edad anciana, cuyo nivel socioeconómico es de clase media.

Como variables para realizar el estudio se excluyeron tanto el sexo como la edad. En cambio, se utilizaron como variable cuantitativa dependiente el número de envases dispensados y como variable cuantitativa independiente los años correlativos desde el año 2000 al 2020.

Las especialidades estupefacientes se han tomado en conjunto sin discernir entre ellas.

Para el recuento, se usó la declaración anual de especialidades estupefacientes, proporcionada por la oficina de farmacia en la que se realizó el estudio.

Para agrupar los datos obtenidos y realizar las tablas y gráficas se utilizó el programa Microsoft Excel. 
CARLOS PÉREZ JIMÉNEZ; PALOMA CARVAJAL GALLARDO; CARMEN GARCÍA MARTÍN EVOLUCIÓN DE LA DISPENSACIÓN DE ESPECIALIDADES FARMACÉUTICAS ESTUPEFACIENTES DESDE EL AÑO 2000 AL 2020 EN LA OFICINA DE FARMACIA SITUADA EN LA PLAZA DEL MERCADO CHICO DE LA CIUDAD DE ÁVILA

\section{Resultados y discusión}

Se realizó un recuento por años de todas las dispensaciones de estupefacientes llevadas a cabo en la farmacia. Dicho conteo queda registrado en la Tabla 1.

TABLA 1: Total de estupefacientes en función del año

\begin{tabular}{|c|c|}
\hline Año & Total de estupefacientes \\
\hline 2000 & 13 \\
\hline 2001 & 38 \\
\hline 2002 & 24 \\
\hline 2003 & 90 \\
\hline 2004 & 93 \\
\hline 2005 & 178 \\
\hline 2006 & 67 \\
\hline 2007 & 113 \\
\hline 2008 & 104 \\
\hline 2009 & 154 \\
\hline 2010 & 153 \\
\hline 2011 & 125 \\
\hline 2012 & 111 \\
\hline 2013 & 137 \\
\hline 2014 & 153 \\
\hline 2015 & 202 \\
\hline 2016 & 316 \\
\hline 2017 & 199 \\
\hline 2018 & 216 \\
\hline 2019 & 262 \\
\hline 2020 & 204 \\
\hline
\end{tabular}


CARLOS PÉREZ JIMÉNEZ; PALOMA CARVAJAL GALLARDO; CARMEN GARCÍA MARTÍN

EVOLUCIÓN DE LA DISPENSACIÓN DE ESPECIALIDADES FARMACÉUTICAS ESTUPEFACIENTES

DESDE EL AÑO 2000 AL 2020 EN LA OFICINA DE FARMACIA SITUADA EN LA PLAZA DEL MERCADO CHICO DE LA CIUDAD DE ÁVILA

Para comenzar con la relación entre los dos factores, se realizó una representación en un diagrama de barras. Los años se representan en el eje de abscisas, al ser la variable independiente. El número de estupefacientes es representado en el eje de ordenadas, al ser la variable dependiente.

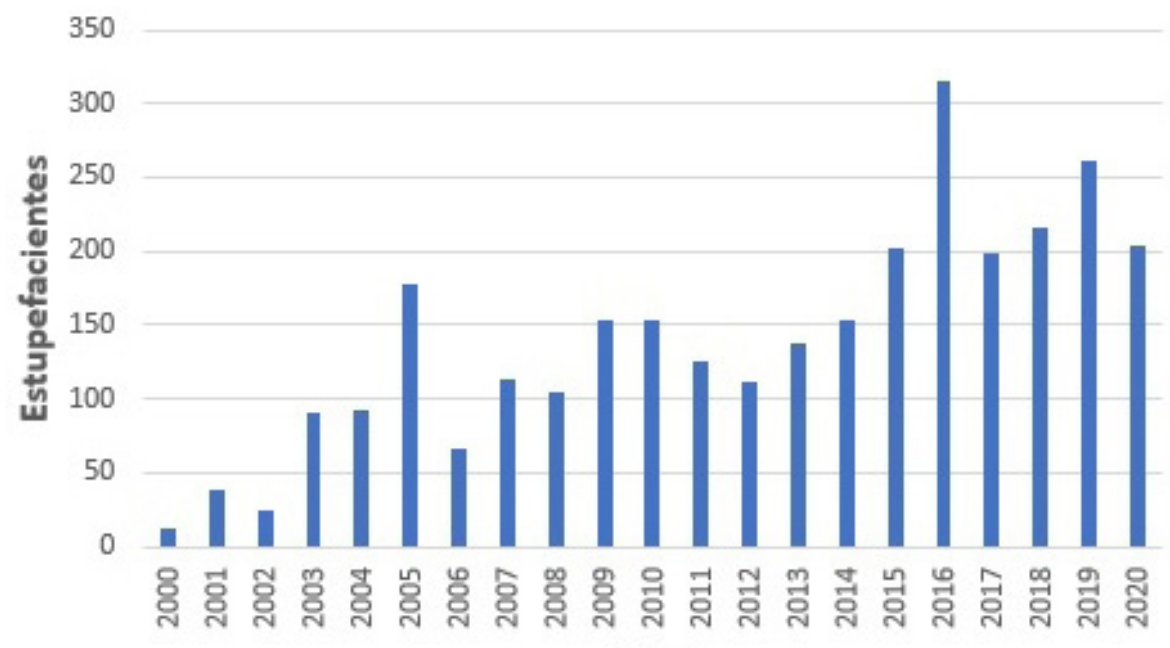

Años

Ilustración 1: Representación de estupefacientes frente a años.

Observando esta representación tampoco se puede establecer ninguna conclusión clara acerca del estudio planteado.

Por ello, para ver cómo es la relación establecida entre nuestras dos variables (años y estupefacientes), se ha realizado un diagrama de dispersión (Ilustración 2). 
CARLOS PÉREZ JIMÉNEZ; PALOMA CARVAJAL GALLARDO; CARMEN GARCÍA MARTÍN EVOLUCIÓN DE LA DISPENSACIÓN DE ESPECIALIDADES FARMACÉUTICAS ESTUPEFACIENTES DESDE EL AÑO 2000 AL 2020 EN LA OFICINA DE FARMACIA SITUADA EN LA PLAZA DEL MERCADO CHICO DE LA CIUDAD DE ÁVILA

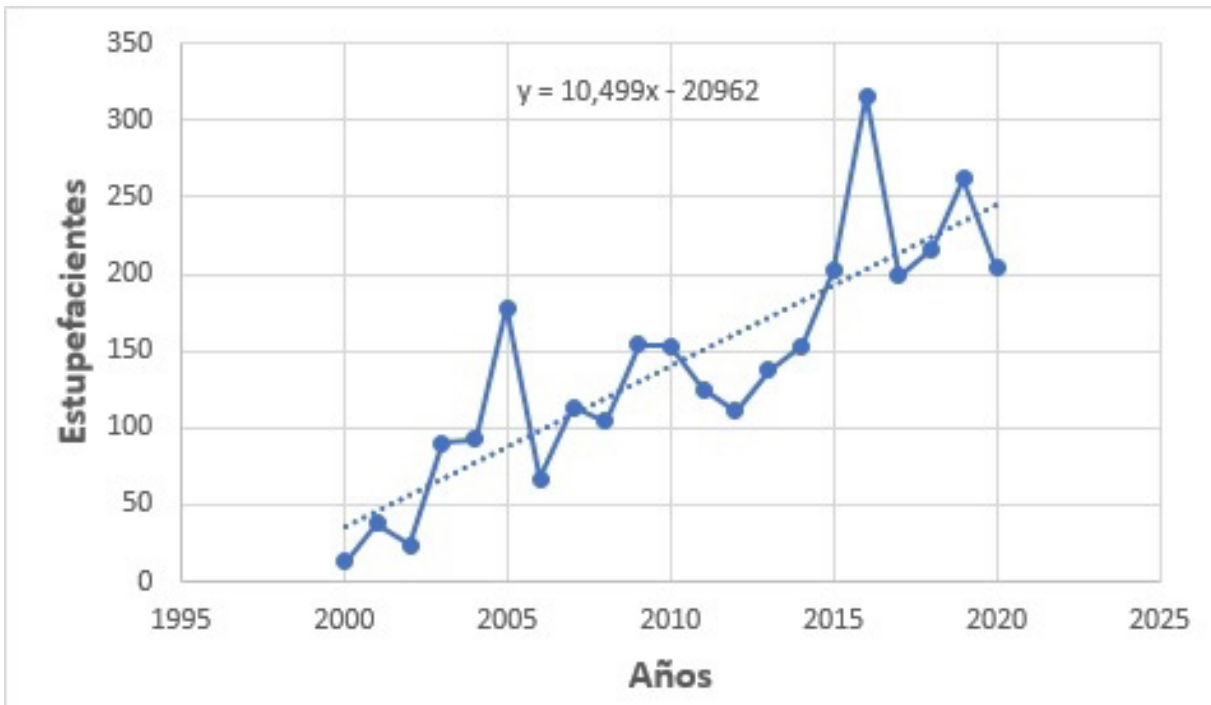

Ilustración 2: Diagrama de dispersión de los estupefacientes en función del año.

Una vez está hecho el diagrama, se saca la recta de la regresión lineal (línea de puntos) para establecer cuál es la tendencia de nuestros datos y también la ecuación de dicha recta.

\section{Conclusión}

Como se comentaba en puntos anteriores, el objetivo de este estudio era comprobar si realmente había una variación en el número de especialidades estupefacientes dispensadas desde año 2000 al 2020 en dicha oficina de farmacia y de qué tipo era esa variación.

Observando la recta de regresión, vemos que a pesar de que la gráfica presenta distintos picos más altos o bajos, en general la tendencia de nuestros datos es creciente. Además, gracias a la ecuación de la recta $(\mathrm{y}=10,499 \mathrm{x}-20962)$ podemos certificar que la pendiente de la recta tiene un valor positivo $(10,499)$, lo que indica también un aumento de los datos.

Por tanto, teniendo en cuenta estos resultados, podemos afirmar que realmente sí que existe una variación en la dispensación de estupefacientes en esta oficina de farmacia. Gracias a la gráfica de regresión y a la línea de tendencia, podemos decir que esta variación es de tipo positivo; es decir, a medida que han ido pasando los 
CARLOS PÉREZ JIMÉNEZ; PALOMA CARVAJAL GALLARDO; CARMEN GARCÍA MARTÍN

EVOLUCIÓN DE LA DISPENSACIÓN DE ESPECIALIDADES FARMACÉUTICAS ESTUPEFACIENTES

DESDE EL AÑO 2000 AL 2020 EN LA OFICINA DE FARMACIA SITUADA EN LA PLAZA DEL MERCADO CHICO DE LA CIUDAD DE ÁVILA

años desde el 2000 al 2020 la dispensación de estupefacientes en la farmacia año tras año ha seguido una tendencia cada vez mayor.

Sin entrar en afirmaciones, hipotéticamente, esta propensión al aumento a lo largo de los años podría estar relacionada con la aparición de nuevos estupefacientes o con la creación de nuevas formas farmacéuticas para estupefacientes ya existentes.

También hipotéticamente, esto podría estar relacionado con un mayor conocimiento sobre la eficacia y utilidad de estas especialidades en distintas enfermedades, como las nombradas en la introducción. Incluso podría ser debido a nuevos estudios que aportan una mayor seguridad a la hora de prescribir distintos tratamientos basados en especialidades estupefacientes.

Estos dos últimos planteamientos únicamente son hipótesis que se podrían tratar de investigar en futuros estudios.

\section{Bibliografía}

Álvarez Y, Farré M. Farmacología de los opioides. Adicción. 2005; 17(2): 21-40.

Consejo General de Colegios Oficiales de Farmacéuticos. Catálogo de medicamentos. Vol. 1. 1. ${ }^{\text {a }}$ ed. Madrid: AEMPS; 2016.

Consumer.healthday.com [Internet]. Consumer Health News; 2017. [citado el 26 de noviembre de 2020]. Disponible en: https://consumer.healthday.com/bone-andjoint-information-4/opioids-990/los-opi-aacute-ceos-y-el-alcohol-son-un-coctelpeligroso-719536.html

Fundacionfemeba.org.ar [Internet]. Fundacion Femeba; 2017. [citado el 23 de noviembre de 2020]. Disponible en: https://www.fundacionfemeba.org.ar/blog/farmacologia-7/ post/cual-es-el-riesgo-de-interaccion-entre-los-opioides-y-los-inhibidores-de-lamonoaminooxidasa-imaos-44911\#: :text=Algunos\%20analg\%C3\%A9sicos\%20opioides \%20est \% C3\% A1n\%20asociados,enzima \%20CYP450\%20por\%20el\%20 IMAO

Info-farmacia.com [Internet]. Info-farmacia; 2018. [citado el 20 de noviembre de 2020]. Disponible en: https://sites.google.com/a/info-farmacia.com/info-farmacia/ultimas-publicaciones/opiaceosrelacionentreestructuraquimicayactividadfarmacologica?mobile=true

Muriel C. Plan Nacional para la Enseñanza y Formación en Técnicas y Tratamiento del Dolor. Vol. 1.1 ed. Salamanca: ARAN; 2001.

News-medical.net [Internet]. News-medical life sciences; 2019. [actualizado el 27 de febrero de 2019; citado el 19 de diciembre de 2020]. Disponible en: https://www. news-medical.net/health/Morphine-Chemistry-(Spanish).aspx

News-medical.net [Internet]. News-medical life sciences; 2019. [actualizado el 27 de febrero de 2019; citado el 27 de noviembre de 2020]. Disponible en: https://www. news-medical.net/health/Opioid-Side-Effects-(Spanish).aspx

Valdivielso SA. Dolor agudo, analgesia y sedación en el niño (IIIa): Farmacocinética y farmacodinamia de los analgésicos opioides. An Esp Pediatr. 1998; 48: 429-440. 
